\title{
Patient-Reported Distress in Myelodysplastic Syndromes and Its Association With Clinical Outcomes: A Retrospective Cohort Study
}

\author{
Jesse D. Troy, PhD, MPH'a Carlos M. de Castro, MD,c; Mary Ruth Pupa, BSNc; Greg P. Samsa, PhD ${ }^{\text {d,e; }}$ \\ Amy P. Abernethy, MD, PhD; ; and Thomas W. LeBlanc, MD, MA, MHS ${ }^{b, c}$
}

\begin{abstract}
Background: NCCN defines distress as a multifactorial, unpleasant emotional experience of a psychological nature that may interfere with patients' ability to cope with cancer symptoms and treatment. Patients with myelodysplastic syndromes (MDS) are at risk for distress due to the largely incurable nature of this hematopoietic malignancy and its symptom burden, yet associations with clinical outcomes are unknown. Methods: We retrospectively reviewed patient-reported distress data from adult ambulatory patients with MDS visiting a single, tertiary care medical center from July 2013 to September 2015. Demographic, diagnostic, treatment, and comorbidity information were abstracted from records along with NCCN Distress Thermometer (DT) and Problem List (PL) scores. Survival was analyzed using the KaplanMeier method and Cox proportional hazards regression. Results: We abstracted 376 DT scores (median, 1; range, 0-10) from 606 visits and 110 patients (median, 2 DT scores/patient; range, 1-16). NCCN Guidelines suggest that patients with DT scores $\geq 4$ should be evaluated for referral to specialty services to address unmet needs. A total of 54 patients (49\%) had at least 1 DT score $\geq 4$ and 20 (18\%) had 2 or more DT scores $\geq 4$; 98 patients (89.1\%) reported 1,379 problems during 23,613 person-days of follow-up (median, 4 problems/patient/visit; range, 1-23). The 5 most frequently reported problems were fatigue (181 times; 78 patients), pain (95 times; 46 patients), worry (80 times; 45 patients), sleep (78 times; 41 patients), and tingling hands/feet (68 times; 33 patients). After adjustment for risk stratification at diagnosis, a single point increase on the DT was associated with an increased risk of death (hazard ratio, 1.18; 95\% Cl, 1.01-1.36). Conclusions: Patients with MDS experience a high burden of distress, and patient-reported distress is associated with clinical outcomes. Distress should be further studied as a prognostic variable and a marker of unmet needs in MDS.
\end{abstract}

Being diagnosed and living with cancer can affect psychological and social well-being, interfere with tasks of daily living, reduce quality of life (QOL), and translate into physical health problems ${ }^{1}$; these psychosocial complications of cancer are referred to as "distress." Approximately one-third of patients with cancer expe-

\footnotetext{
From the aDivision of Blood and Marrow Transplantation, Department of Pediatrics, and bivision of Hematological Malignancies and Cellular Therapy, Department of Medicine, Duke University School of Medicine; 'Duke Cancer Institute; dDepartment of Biostatistics and Bioinformatics, Duke University School of Medicine, Durham, North Carolina; and 'Data, Informatics and Statistics Core, Palliative Care Research Cooperative, Durham, North Carolina; and fFlatiron Health, New York, New York. Submitted June 29, 2017; accepted for publication October 9, 2017. Dr. Troy has disclosed that he has is a consultant for Gamida, and has received grants from Seattle Genetics. Dr. Abernethy has disclosed that she is employed at Flatiron Health, is on the Board of Directors for Athena Health, and owns stock in Flatiron Health and Athena Health. Dr. LeBlanc has disclosed that he is a consultant for Celgene, Boehringer Ingelheim, Pfizer, Epi-Q/Janssen, Flatiron Health, and Helsinn; and that
}

rience distress. ${ }^{3}$ Distress may affect successful management of cancer through negatively impacting patients' adherence to therapy and decision-making capacity, and distress has been associated with reduced survival in some cancers. ${ }^{4-6}$

Myelodysplastic syndromes (MDS) are diagnosed primarily in older people and represent a diverse group

he has received grants from Seattle Genetics, AstraZeneca, and Cambia Health Solutions. The remaining authors have disclosed that they have no financial interests, arrangements, affiliations, or commercial interests with the manufacturers of any products discussed in this article or their competitors.

Author contributions: Study concept and design: Troy, de Castro, Samsa, Abernethy, LeBlanc. Data acquisition: Troy, Pupa, LeBlanc. Data analysis: Troy. Initial manuscript draft: Troy. Critical revision of statistical analyses: Samsa. Data interpretation: All authors. Writing of manuscript: All authors.

Correspondence: Thomas W. LeBlanc, MD, MA, MHS, Duke University School of Medicine, Department of Medicine, Division of Hematological Malignancies and Cellular Therapy, Box 2715, Durham, NC 27710.

E-mail: thomas.leblanc@duke.edu 
Troy et al

of malignant bone marrow disorders with variable clinical outcome. ${ }^{7} \mathrm{QOL}$ is substantially impacted by MDS. ${ }^{8}$ Distress is increased in patients with cancer when comorbid conditions are present, symptoms are uncontrolled, and changes occur in disease status. $^{2}$ Thus, distress is likely to be a concern with respect to clinical outcomes in MDS.

The NCCN Distress Thermometer (DT) and accompanying Problem List (PL) have been used to measure distress in several tumor types but has not yet been applied to MDS. Although MDS cases were included in studies describing distress in patients receiving hematopoietic stem cell transplant (HSCT), these studies did not report results for MDS specifically. ${ }^{9-11}$ Furthermore, because HSCT is infrequently used to treat MDS, these results are not likely to be representative of distress levels in the broader MDS population.

To our knowledge, no data have been published on distress in patients with MDS receiving nontransplant therapies in an ambulatory care setting, where most of these patients are managed. There is also reason to suspect that patient-reported distress might correlate with disease activity, given that it reflects how patients are feeling, perhaps beyond what is measurable in routine laboratory assessments (eg, fatigue is known to correlate poorly with hemoglobin levels). ${ }^{12}$ Therefore, we conducted a retrospective evaluation of distress in patients with MDS. Our intent was to describe the magnitude and sources of distress, and explore associations with clinical outcomes among patients with MDS.

\section{Methods}

\section{Patients}

Eligible patients were aged $\geq 18$ years with pathologically confirmed MDS (ICD-9 codes 238.72-238.75) and visited our tertiary care outpatient clinics between July 2013 and September 2015. The start date for this study reflects the date that Duke Cancer Institute began using a new electronic medical record (EMR) system in which the DT and PL responses were entered as part of usual care. Patients were excluded if they received HSCT within 100 days of their first clinic visit during the data abstraction period.

Our initial search of the EMR identified $248 \mathrm{pa}$ tients who were then screened. We excluded 124 patients (50\%): 27 were visiting our clinics for other malignancies, 21 had nonmalignant disease, 28 had MDS but visited our clinics for HSCT, 26 had a myeloproliferative neoplasm or an "overlap syndrome," 11 had insufficient information to confirm an MDS diagnosis, and 11 patients had MDS but no record of DT or PL responses.

Therefore, we abstracted data for 124 eligible patients who contributed PL responses only $(\mathrm{N}=14)$, or both DT and PL responses $(\mathrm{N}=110)$. The study was reviewed and approved by the Duke University School of Medicine Institutional Review Board, and a consent waiver was obtained before data abstraction.

\section{Patient-Reported Distress}

Distress is evaluated using the NCCN DT as part of routine care for all patients seen at Duke Cancer Institute. The DT is a patient-reported screening tool that measures patients' distress during the previous week using an 11-point Likert scale ( $0=$ no distress, 10 = extreme distress), and is accompanied by a 39-item PL that allows patients to indicate the source of their distress in 5 different domains using yes/no answers. The DT is a valid measure of distress, with a score of 4 identifying potentially actionable distress as described in NCCN Guidelines algorithms for psychological/ psychiatric treatment. ${ }^{2}$ At Duke Cancer Institute, patients with MDS are asked to complete the DT and PL on paper at outpatient visits, which is then transcribed into the EMR by clinic staff at the point of care. We abstracted data for eligible patients at any visit documented in the EMR during our study period.

\section{Demographic, Disease, and Comorbidity Information}

We abstracted the following from the EMR: age, sex, race, ethnicity, insurance status, marital status, cancer history, month/year of diagnosis, pathologic subtype, and risk stratification at diagnosis. Patients were designated as low, intermediate, or high risk using the original or revised International Prognostic Scoring System (IPSS/IPSS-R; N=79) or the WHO Prognostic Scoring System $(\mathrm{N}=45)$. We used both risk stratification schemas because many charts did not include all of the necessary data elements to calculate risk by the IPSS/IPSS-R. We evaluated the validity of this approach by comparing Kaplan-Meier survival curves across risk strata and observed the expected pattern of decreasing survival with increasing risk (not shown). The Charlson comorbidity index 
Patient-Reported Distress in MDS

(CCI) was used to describe comorbidities present at diagnosis using data from the EMR.

\section{Clinical Outcomes}

Data on the following outcomes were abstracted, when available, from each clinical encounter: Karnofsky or ECOG performance status, current and newly added or changed MDS therapy (hypomethylating agent, lenalidomide, growth factors, iron chelation, transfusions, other chemotherapy [eg, induction therapy], and HSCT), blood counts, participation in a clinical trial, and progression to acute myeloid leukemia (AML). Vital status was determined through review of the EMR and verified by our institution's tumor registrar.

\section{Statistical Analysis}

The primary objective of our inferential analyses was to identify differences in the reporting of potentially actionable distress across a range of patient and disease characteristics. Thus, when patients reported $>1$ DT score, they were classified according to their maximum score. Number of observations was not associated with the maximum-reported DT score (not shown). Nonparametric tests were used to compare DT scores between subgroups. Survival associated with DT score was estimated using the KaplanMeier method. Follow-up time was calculated from the date of study enrollment to the date of death or last visit during our study period. We also conducted stepwise, left-truncated Cox proportional hazards regression modeling to predict survival from time of diagnosis based on repeated measures of distress observed in our study. We considered as covariates only factors that we felt, based on previous research, might be associated with survival: age at diagnosis, use of therapy (hypomethylating agents/lenalidomide/growth factors/transfusions/chelation), CCI score, risk stratification at diagnosis, ever-report of fatigue, and frequency of fatigue. Analyses were conducted using SAS 9.4 (SAS Institute, Cary, NC).

\section{Results}

Table 1 shows characteristics of the 124 included patients. The median age at diagnosis was 70 years, most were white $(74.2 \%)$ or male $(66.9 \%)$, and $29.0 \%$ selfreported a prior history of cancer other than basal/squamous cell skin cancers ( 40 cancers reported among 36

\begin{tabular}{|c|c|}
\hline & $\begin{array}{l}\text { Patients, } N(\%) \\
\quad(\mathrm{N}=124)\end{array}$ \\
\hline \multicolumn{2}{|l|}{ Age at diagnosis, y } \\
\hline Mean (SD) & $67.8(10.6)$ \\
\hline Median (range) & $69.79(30.68-88.18)$ \\
\hline \multicolumn{2}{|l|}{ Months from diagnosis to first visit } \\
\hline Mean (SD) & $21.1(32.8)$ \\
\hline Median (range) & $5.88(0-206.7)$ \\
\hline \multicolumn{2}{|l|}{ Sex } \\
\hline Male & $83(66.9 \%)$ \\
\hline Female & $41(33.1 \%)$ \\
\hline \multicolumn{2}{|l|}{ Race } \\
\hline White & $92(74.2 \%)$ \\
\hline Nonwhite & $11(8.9 \%)$ \\
\hline Unknown & $21(16.9 \%)$ \\
\hline \multicolumn{2}{|l|}{ Marital status } \\
\hline Not married & $20(16.1 \%)$ \\
\hline Married & $84(67.7 \%)$ \\
\hline Unknown & $20(16.1 \%)$ \\
\hline \multicolumn{2}{|l|}{ Health insurance } \\
\hline Medicare & $87(70.2 \%)$ \\
\hline Medicaid & $3(2.4 \%)$ \\
\hline Private payer & $30(24.2 \%)$ \\
\hline Other & $4(3.2 \%)$ \\
\hline \multicolumn{2}{|l|}{ MDS subtype } \\
\hline Refractory anemia & $9(7.3 \%)$ \\
\hline $\begin{array}{l}\text { Refractory cytopenia with unilineage } \\
\text { dysplasia }\end{array}$ & $2(1.6 \%)$ \\
\hline $\begin{array}{l}\text { Refractory cytopenia with multilineage } \\
\text { dysplasia }\end{array}$ & $42(34.1 \%)$ \\
\hline Refractory anemia with ring sideroblasts & $16(13.0 \%)$ \\
\hline Refractory anemia with excess blasts & $43(35.0 \%)$ \\
\hline MDS associated with del(5q) & $7(5.7 \%)$ \\
\hline MDS, unclassifiable & $3(2.4 \%)$ \\
\hline Unknown & $1(0.8 \%)$ \\
\hline \multicolumn{2}{|l|}{ Risk stratification ${ }^{a}$} \\
\hline Low & $60(48.4 \%)$ \\
\hline Intermediate & $26(21.0 \%)$ \\
\hline High & $38(30.6 \%)$ \\
\hline \multicolumn{2}{|l|}{ Cancer history } \\
\hline No & $88(71.0 \%)$ \\
\hline Yes & $36(29.0 \%)$ \\
\hline \multicolumn{2}{|l|}{ Died during study period } \\
\hline Yes & $25(20.2 \%)$ \\
\hline No & $99(79.8 \%)$ \\
\hline \multicolumn{2}{|l|}{ Progressed to AML } \\
\hline No & $119(96.0 \%)$ \\
\hline Yes & $5(4.0 \%)$ \\
\hline
\end{tabular}

Abbreviations: AML, acute myeloid leukemia; MDS, myelodysplastic syndromes.

aRisk stratification is based on the original and revised International Prognostic Scoring System (IPSS/IPSS-R) where available. For patients with unknown IPSS/IPSS-R ( $\mathrm{N}=45$ ), the WHO Prognostic Scoring System was used based on information obtained from the diagnosis visit. 
patients). Approximately half (48.4\%) had low-risk disease, and 5 (4.0\%) experienced disease progression to AML. Predominant pathologic subtypes were refractory anemia with excess blasts $(35.0 \%)$, refractory cytopenia with multilineage dysplasia (34.1\%), and refractory anemia with ring sideroblasts (13.0\%). Healthcare encounters were generally uncommon (Figure 1 ). Only 25 patients (20.2\%) had $\geq 1$ hospitalizations and $12.1 \%$ visited the emergency department at least once. Among the 25 patients who died during the study period, only 2 used hospice, and we did not find evidence of referral to palliative care clinics for any included patients.

\section{Patient-Reported Distress}

We abstracted 376 DT scores (median, 1; interquartile range [IQR], 0-3.5; range, 0-10) from 606 visits and 110 patients (median, 2 DT scores/patient; IQR, 1-4; range, 1-16). A total of 54 patients had at least 1 DT score $\geq 4$ and 20 had 2 or more DT scores $\geq 4$. When patients were classified by their maximum DT score (median, 3; IQR, 1-7; range, 0-10), ever-use of therapy was associated with greater maximum distress ( $\mathrm{N}=75$; median, 4; IQR, 1-8; range, 0-10) compared with no therapy $(\mathrm{N}=35$; median, 2; IQR, 1-4; range, $0-9 ; P=.01)$. Ever-use of hypomethylating agents $(P=.75)$ or lenalidomide $(P=.09)$ was not associated with distress. However, receipt of growth factors was associated with higher maximum distress $(\mathrm{N}=32$; me-

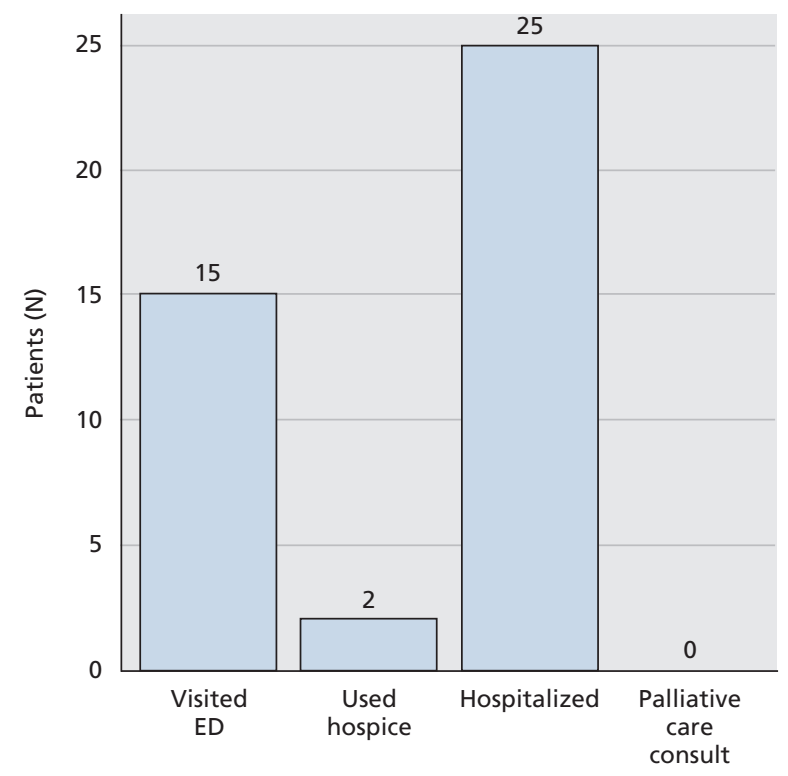

Figure 1. Healthcare utilization. Abbreviation: ED, emergency department. dian, 5.5; IQR, 2-8; range, 0-10) than never receiving growth factors $(\mathrm{N}=78$; median, 2.5; IQR, 1-6; range, $0-10 ; P=.02)$. Receipt of red blood cells was associated with higher maximum distress $(\mathrm{N}=54$; median, 4.5; IQR, 1-8; range, 0-10) compared with never receiving packed red cells $(\mathrm{N}=56$; median, 2.5; IQR, 1-5; range, $0-10 ; P=.03)$. Patients who received platelets reported higher maximum distress $(\mathrm{N}=22$; median, 6 ; IQR, 3-8; range, 0-10) compared with those who did not $(\mathrm{N}=88$; median, 2.5 ; IQR, 1-6; range, $0-10)$; this difference approached statistical significance $(P=.06)$. Iron chelation was unrelated to distress, although very few patients in our cohort were chelated $(\mathrm{N}=6$; $P=.98)$. The number of visits in which patients underwent each of these therapies was not related to the maximum-reported DT score. Finally, we did not observe any association between the maximum-reported DT score and age at diagnosis, sex, race, marital status, type of health insurance, prior history of cancer, CCI score, number of visits per patient during the study period, MDS subtype, risk stratification at diagnosis, being hospitalized, or visiting the emergency department ( $P>.05$ for all).

\section{Sources of Distress}

The most frequently reported categories of problems were physical (233 reports), emotional (104 reports), practical (49 reports), and family (41 reports). Spiritual problems were reported only once during the study. Within each of these categories, the number of problems reported per patient was not strongly associated with DT scores.

We analyzed the association between the DT and PL in the 110 patients who reported both. A total of 98 of these patients $(89.1 \%)$ reported 1,379 problems during 23,613 person-days of follow-up (median, 4 problems/patient/visit; range, $1-23$ ). The 5 most frequent problems were fatigue (181 times; 78 patients), pain (95 times; 46 patients), worry (80 times; 45 patients), sleep (78 times; 41 patients), and tingling hands/feet (68 times; 33 patients) (Figure 2 ). The total number of problems reported per patient was significantly correlated with the maximum distress level reported by each patient $\left(\mathrm{r}_{\text {Spearman }}=0.70\right.$; 95\% CI, 0.59-0.78; P<.0001).

\section{Patient-Reported Distress and Survival}

A maximum-reported DT score $\geq 4$ was associated with poor survival in our Kaplan-Meier analy- 


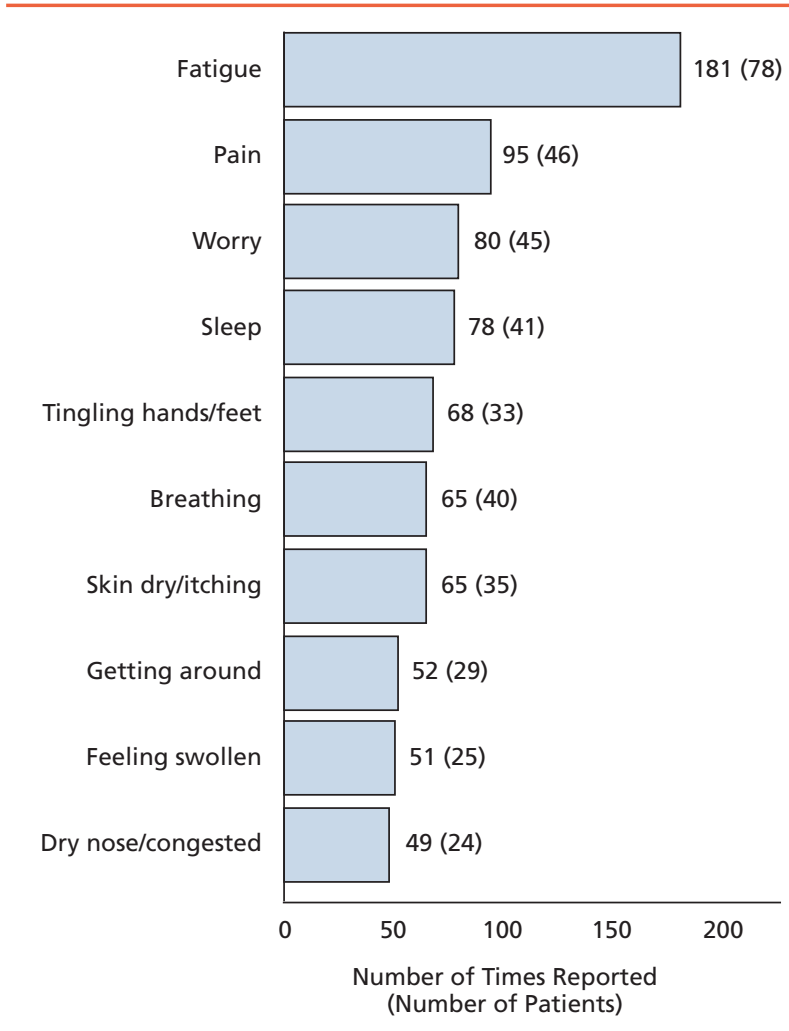

Figure 2. Top 10 most frequently reported problems.

sis (Figure $3 ; P=.01$ ). Median time to death in patients with a DT score $<4$ was 9.9 months (range, $3.8-22.0$; 5 deaths) compared with a median of 5.1 months (range, 0.5-20.8) in patients reporting a maximum DT $\geq 4$ (16 deaths). Cox proportional hazards regression models showed the DT, prognostic strata at diagnosis, and ever-use of therapy to be significant predictors of death (Table 2). Only the DT and prognostic scores remained significant after backward elimination. In the final model, each additional point on the DT scale was associated with an $18 \%$ increase in risk of death (95\% CI, 1.01-1.36) after controlling for risk stratification at diagnosis.

\section{Discussion}

We observed 3 important findings in this retrospective cohort study of patient-reported distress in ambulatory patients with MDS visiting a Comprehensive Cancer Center. First, these patients have a relatively high burden of distress, with physical symptoms representing the most frequently reported issue, and a greater overall symptom burden in these patients was associated with higher distress. Second,

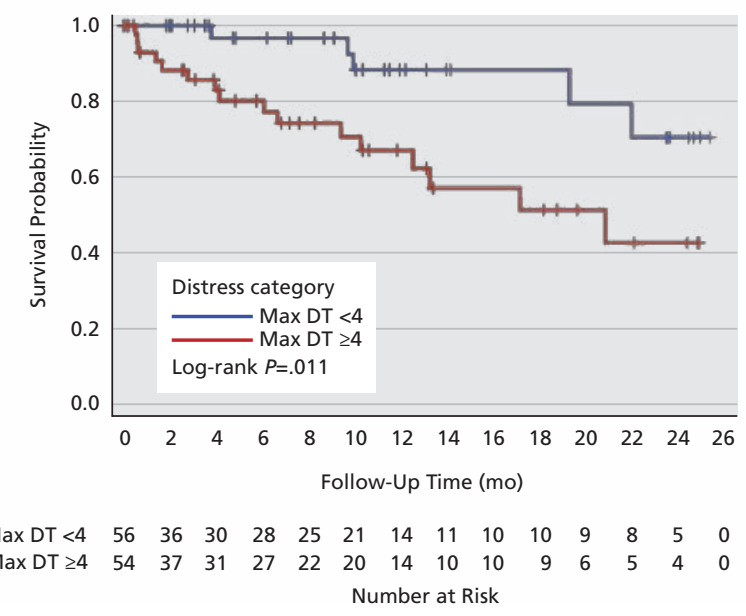

Figure 3. Overall survival by maximum reported distress. Abbreviations: DT, Distress Thermometer; Max, maximum.

distress is associated more with the receipt of treatments and services, such as blood transfusions, than with MDS prognosis, demographic variables, or comorbidities. Lastly, distress scores also appear to be related to overall survival, even after controlling for MDS prognosis.

Patients in our cohort were markedly distressed. We found that 54 of 110 patients (49\%) had at least one DT score of 4 during the study period. However, the median DT across all patients and visits in our study was 1 , and covered the entire range of the scale from 0 to 10 . This suggests that patients with MDS are not consistently distressed, but have a high probability of experiencing at least one clinical encounter characterized by marked distress and unmet needs in the course of 1 or 2 years of observation. We did not find other studies with longitudinal evaluations of distress in cancer for direct comparison. However, one study estimated that $33 \%$ of patients with cancer experience distress levels of $\geq 4$ on the DT before their first outpatient appointment with an oncologist ${ }^{13}$; this is similar to the $35 \%$ of our MDS cohort who rated their distress as $\geq 4$ within 60 days after diagnosis.

Our results suggest that the distress experienced by patients with MDS is related to physical symptoms, particularly fatigue and pain, and psychological issues such as worry. These sources of distress mirror the results of a large survey, which reflected a marked prevalence of fatigue and other physical symptoms in patients with MDS, including bruising, night sweats, and bone pain. ${ }^{14}$ However, there may be differences between the prevalence of symp- 
Troy et al

\begin{tabular}{|c|c|c|c|c|}
\hline Covariate & AIC With Covariates & AIC Change $^{a}$ & HR $(95 \% \mathrm{Cl})$ & $P$ Value \\
\hline \multicolumn{5}{|l|}{ Univariable models } \\
\hline Distress thermometer ${ }^{b}$ & 97.38 & -9.56 & $1.28(1.11-1.47)$ & $<.001$ \\
\hline Age at diagnosis ${ }^{\mathrm{b}}$ & 106.70 & -0.24 & $1.04(0.99-1.09)$ & .135 \\
\hline Risk stratification at diagnosisc & 96.24 & -10.70 & $6.92(2.30-20.79)$ & $<.001$ \\
\hline Charlson comorbidity index score ${ }^{b}$ & 107.34 & 0.40 & $1.18(0.93-1.48)$ & .206 \\
\hline Use of therapy ${ }^{d}$ & 104.48 & -2.46 & $3.99(0.89-17.87)$ & .035 \\
\hline Reported fatigue $^{d}$ & 108.27 & 1.33 & $1.58(0.51-4.91)$ & .414 \\
\hline Frequency of fatigue $^{b}$ & 107.46 & 0.52 & $0.89(0.73-1.09)$ & .224 \\
\hline \multicolumn{5}{|l|}{ Final model } \\
\hline Distress thermometer ${ }^{b}$ & \multirow{2}{*}{93.53} & \multirow{2}{*}{-13.41} & $1.18(1.01-1.36)$ & .030 \\
\hline Risk stratification at diagnosis ${ }^{c}$ & & & $4.02(1.26-12.83)$ & .016 \\
\hline
\end{tabular}

Abbreviations: AIC, Akaike information criteria; $\mathrm{HR}$, hazard ratio.

aAIC change is calculated from the AIC of the model without covariates, 106.94.

bSingle-point increase.

cHigh vs intermediate/low.

dYes vs no.

toms and their contribution to distress. For example, many patients with MDS have reported bruising in symptom surveys, ${ }^{14}$ but this does not mean that bruising is necessarily a distressing symptom for most patients. The distinction between symptom prevalence and contribution to distress has implications for the identification of unmet needs and development of interventions to improve patient experiences.

We observed that reporting of problems in specific areas (eg, physical problems) was not associated with the maximum distress level. However, the total number of problems reported was associated with maximum-reported DT score. This suggests that the overall burden of problems, rather than problems in any specific area, is most important. It is also worth noting that despite the burden of problems reported in our study, we found no evidence of referrals to palliative care services in the EMR, although these services were available at our center during the study period. This observation is consistent with the general underuse of palliative care services that has been noted in hematologic malignancies relative to solid tumor cancers. ${ }^{15}$ Literature on the use of palliative care services in MDS is scant. A recent review ${ }^{16}$ suggested that possible reasons for the divide between palliative care needs and medical practice in MDS may include clinical features of MDS that complicate recognizing end-stage disease, misunderstanding of the intent or appropriateness of palliative care by hematologists in general, or misunderstanding of MDS treatment efficacy by both patients and clinicians.
We also found an association between receipt of certain services and overall level of distress. For example, patients receiving blood or platelet transfusions were more distressed than others. Interestingly, however, we did not find such associations with MDS risk scores or other traditional variables associated with clinical outcomes, such as age or comorbidities. These results suggest that patient-reported distress is most closely associated with patient encounters with (and use of) the healthcare system rather than with classical predictors of risk in MDS. This implies that patient-reported distress adds meaningful information not reflected in traditional clinical variables. The specificity of this finding argues against the possibility of bias arising from the study design; for example, that patients receiving transfusions simply had more frequent visits and thus more opportunities to report distress. In that eventuality, we would have expected to see an association between distress and chemotherapy, which we did not. Therefore, patient-reported distress should be studied further as a potential signal for unmet needs or as a prognostic variable.

Finally, using serial measures of distress in our cohort, we observed that greater distress, regardless of when it is reported during routine clinical followup, is associated with increased risk of death even after controlling for the most important prognostic factor in MDS: risk score. This interesting finding adds to the literature suggesting that patientreported symptom data offer important prognostic information beyond traditional clinical data, such 
as the recent description of fatigue as an independent predictor of survival in MDS. ${ }^{17}$ Our study was limited in its ability to explore this finding further due to the small number of deaths in our cohort. A larger, multisite investigation of this association is warranted.

The primary limitation of our study is its retrospective nature. In particular, the number of visits per patient and the elapsed time between visits were determined by each patient's medical needs rather than per-protocol. Therefore, although our study included longitudinal assessment of distress, our cohort was not measured from a common baseline. However, we were able to leverage the longitudinal data for estimating the association of distress with survival using well established statistical methods. Also, we did not validate DT findings against other measures, such as the Hospital Anxiety and Depression Scale, as has been done in other tumor types. Nonetheless, approximately one-half of the patients in our study reported DT scores greater than or equal to the NCCN-defined threshold of 4, and reporting a maximum DT at this level was associated with the use of several diseasespecific therapies, offering some face validity for the NCCN-defined distress threshold in MDS.

\section{Conclusions}

Despite these limitations, our study offers what we believe to be the first inventory of distress in patients with MDS regularly attending an outpatient clinic. Given that most patients are ambulatory, our study likely represents the usual clinical experience in MDS. Most importantly, our study demonstrates the ability of the NCCN DT to measure distress in patients with MDS, and that these distress levels may have deleterious effects on aspects of constitutional and social health. Therefore, our results indicate that the DT is a potential screening tool for identifying patients with MDS who require intervention for treatment of distress. This observation is well-timed given the recent development of MDS-specific measures that might be used as end points in studies examining the efficacy of interventions for improving QOL. ${ }^{18}$

In summary, distress is common in patients with MDS and is associated with physical, emotional, and social problems. It may be exacerbated by use of MDS-specific therapies and is potentially associated with survival. Our findings show that distress can be measured in ambulatory patients with MDS using the NCCN DT. Future studies should evaluate the efficacy of interventions to improve distress and ameliorate patient-reported problems in MDS.

\section{References}

1. VanHoose L, Black LL, Doty $K$, et al. An analysis of the distress thermometer problem list and distress in patients with cancer. Support Care Cancer 2015;23:1225-1232.

2. Holland JC, Deshields TL, Andersen B, et al. NCCN Clinical Practice Guidelines in Oncology: Distress Management. Version 1.2018. Accessed January 31,2018 . To view the most recent version of these guidelines, visit NCCN.org.

3. Zabora J, BrintzenhofeSzoc K, Curbow B, et al. The prevalence of psychological distress by cancer site. Psychooncology 2001;10:19-28.

4. Partridge AH, Wang PS, Winer EP, Avorn J. Nonadherence to adjuvant tamoxifen therapy in women with primary breast cancer. J Clin Oncol 2003;21:602-606.

5. Brown KW, Levy AR, Rosberger Z, Edgar L. Psychological distress and cancer survival: a follow-up 10 years after diagnosis. Psychosom Med 2003;65:636-643.

6. Pirl WF, Greer JA, Traeger L, et al. Depression and survival in metastatic non-small-cell lung cancer: effects of early palliative care. J Clin Oncol 2012;30:1310-1315.

7. Troy JD, Atallah E, Geyer JT, Saber W. Myelodysplastic syndromes in the United States: an update for clinicians. Ann Med 2014;46:283-289.

8. Heptinstall K. Quality of life in myelodysplastic syndromes. A special report from the Myelodysplastic Syndromes Foundation, Inc. Oncology (Williston Park, NY) 2008;22(2 Suppl Nurse Ed):13-18; discussion 19.

9. Bevans $M$, Wehrlen L, Prachenko O, et al. Distress screening in allogeneic hematopoietic stem cell (HSCT) caregivers and patients. Psychooncology 2011;20:615-622.

10. Braamse AM, van Meijel B, Visser O, et al. Distress, problems and supportive care needs of patients treated with auto- or allo-SCT. Bone Marrow Transplant 2014;49:292-298.

11. Ransom S, Jacobsen PB, Booth-Jones M. Validation of the Distress Thermometer with bone marrow transplant patients. Psychooncology 2006;15:604-612.

12. Efficace F, Gaidano G, Breccia M, et al. Prevalence, severity and correlates of fatigue in newly diagnosed patients with myelodysplastic syndromes. $\mathrm{Br}$ J Haematol 2015;168:361-370.

13. Kendall J, Glaze K, Oakland S, et al. What do 1281 distress screeners tell us about cancer patients in a community cancer center? Psychooncology 2011;20:594-600.

14. Steensma DP, Heptinstall KV, Johnson VM, et al. Common troublesome symptoms and their impact on quality of life in patients with myelodysplastic syndromes (MDS): results of a large internet-based survey. Leuk Res 2008;32:691-698.

15. LeBlanc TW. Addressing end-of-life quality gaps in hematologic cancers: the importance of early concurrent palliative care. JAMA Intern Med 2016;176:265-266.

16. Nickolich M, El-Jawahri A, LeBlanc TW. Palliative and end-of-life care in myelodysplastic syndromes. Curr Hematol Malig Rep 2016;11:434-440.

17. Efficace F, Gaidano G, Breccia M, et al. Prognostic value of self-reported fatigue on overall survival in patients with myelodysplastic syndromes: a multicentre, prospective, observational, cohort study. Lancet Oncol 2015;16:1506-1514.

18. Abel GA, Efficace F, Buckstein RJ, et al. Prospective international validation of the Quality of Life in Myelodysplasia Scale (QUALMS). Haematologica 2016;101:781-788. 